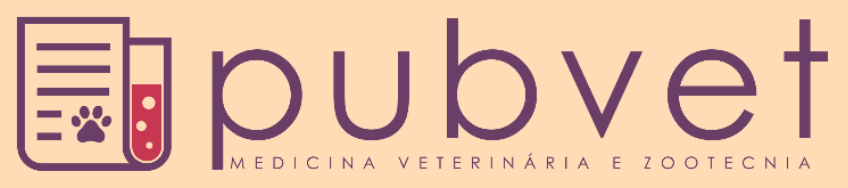

https://doi.org/10.31533/pubvet.v15n12a984.1-5

\title{
Importância da homeopatia no tratamento paliativo da doença renal crônica: Estágio 4
}

\author{
Anderson Scherer ${ }^{1} \bullet$, Francielly Santos Mendes $^{2 \ominus}$, Igor Silva Silito ${ }^{2} \bullet$, Renata Tesser Rocha $^{3 *} \bullet$ \\ ${ }^{1}$ Professor da Universidade Anhembi Morumbi, Comunicação Clínica e Projeto de Integração Saúde e Comunidade, São Paulo - SP Brasil. \\ ${ }^{2}$ Bacharelando em Medicina Veterinária na Universidade Anhembi Morumbi (UAM). São Paulo, SP - Brasil. \\ ${ }^{3}$ Professor da Universidade Anhembi Morumbi, Fisioterapia e reabilitação animal, São Paulo - SP Brasil. \\ *Autor para correspondência, E-mail: igorsilito20@gmail.com
}

\begin{abstract}
Resumo. Foi realizado um levantamento bibliográfico a respeito do uso da homeopatia aplicada ao alívio da dor e do sofrimento em animais acometidos com Doença renal crônica em estágio 4. Uma revisão bibliográfica em livros, periódicos, artigos científicos e revistas eletrônicas que julgamos pertinentes. A História da homeopatia, desde seu surgimento, sofreu grandes contribuições, como de Hipócrates, conhecido como "o pai da medicina" e Christian Friedrich Samuel Hahnemann, médico Alemão, que no século XIX, concretizou a homeopatia como a conhecemos hoje. Diferentemente da alopatia convencional, a homeopatia se mostra uma alternativa viável para findar e/ou minimizar a dor do animal. Comum na Clínica de pequenos animais, a Doença Renal Crônica, patologia caracterizada pela destruição dos néfrons até o ponto de perda de função renal, com irreversibilidade do processo e TFG inferior a 50\%, pode ser estadeada baseando-se em parâmetros da concentração de creatinina. De acordo com a literatura consultada, fica claro que o tratamento alopático de eleição, apesar de contribuir no controle dos sinais clínicos manifestados, é incapaz de garantir a cura, tampouco a irreversibilidade do processo. Assim, a homeopatia se apresenta como uma alternativa para o cuidado paliativo do animal, garantindo a diminuição de riscos e a melhora na qualidade de vida do animal.
\end{abstract}

Palavras-chave: Doença renal crônica, homeopatia, tratamento paliativo

\section{Importance of homeopathy in the palliative treatment of chronic kidney disease: Stage 4}

Abstract. A bibliographical survey was carried out regarding the use of homeopathy applied to the relief of pain and suffering in animals affected with stage 4 chronic kidney disease. A bibliographic review in books, periodicals, scientific articles and electronic journals that we consider relevant. The history of homeopathy, since its inception, has undergone great contributions, such as Hippocrates, known as "the father of medicine" and Christian Friedrich Samuel Hahnemann, a German physician, who in the 19th century, concretized homeopathy as we know it today. Unlike conventional allopathy, homeopathy proves to be a viable alternative to end and/or minimize the animal's pain. Common in small animal clinics, Chronic Kidney Disease, a pathology characterized by the destruction of nephrons to the point of loss of renal function, with irreversibility of the process and GFR lower than 50\%, can be staged based on creatinine concentration parameters. According to the literature consulted, it is clear that the allopathic treatment of choice, despite contributing to the control of clinical signs manifested, is incapable of guaranteeing a cure, nor the irreversibility of the process. Thus, homeopathy presents itself as an alternative for the palliative care of the animal, ensuring the reduction of risks and improvement in the animal's quality of life.

Keywords: Chronic kidney disease, homeopathy, palliative treatment 


\section{Introdução}

Indícios apontam que a homeopatia tenha surgido no antigo Egito (Costa et al., 2009). Com o passar dos anos, foi-se espalhando pelos continentes, chegando até as civilizações da América, Incas, Astecas e Nativos americanos. Mas a concepção inicial da homeopatia surgiu em meados do século IV A.C. com Hipócrates, o maior médico da antiguidade, conhecido como o pai da medicina. Ele mencionava que "tudo acontece conforme a natureza" e que "ela tem o poder de curar", com isso, ele acreditava que o tratamento de doenças consiste em três pilares centrais (Pereira, 2012).

O primeiro princípio que rege a homeopatia é que a natureza é capaz de restabelecer a saúde do doente e, por isso, o médico deve imitar a patologia durante o tratamento para que seu paciente volte às condições fisiológicas. Já o segundo princípio geral afirma que sintomas podem ser tratados de formas diferentes do seu acontecimento. Por fim, o terceiro princípio diz que a doença pode ser neutralizada, por medidas relativas a ela, ou seja, a doença pode ser curada por substâncias que mimetizem os sintomas, chamada Lei dos Semelhantes (Pereira, 2012). Para essa corrente de pensamento, a patologia é uma perturbação da energia vital, sendo a homeopatia responsável por restabelecer o equilíbrio. O que difere a alopatia da homeopatia é que a primeira está preocupada com a doença, enquanto a outra tenta um equilíbrio para diminuir a susceptibilidade às patologias, ou seja, preocupa-se com a saúde (Bear \& Bellucco, 2006).

A homeopatia humana como se conhece, foi criada e concretizada há duzentos anos, por Christian Friedrich Samuel Hahnemann, médico Alemão. Em 1843, Hahnemann curou o seu cavalo por meio da homeopatia e anunciou: "se as leis que proclamo são as da Natureza, elas serão válidas para todos os seres vivos". Assim, dá-se o início da homeopatia veterinária, cujas técnicas e métodos vêm sendo aprimorados desde então (Pires, 2005; Souza, 2002).

No Brasil, a Homeopatia teve uma grande ampliação em 1942, devido à publicação do Dr. Nilo Cairo "Guia Prático da Veterinária Homeophatica" ou "Tratamento homeopático de todas as moléstias dos animais domésticos". Logo após, nos anos 50, o Dr. Cláudio Real, que estudou homeopatia na França, se tornou o primeiro brasileiro homeopata animal. Mas só em 1996, a Medicina Homeopata Veterinária foi validada pelo Conselho Federal de Medicina Veterinária (Pires, 2005).

"HOMEOPATIA é uma palavra de origem grega: HOMEOS - semelhante, da mesma natureza, igual, análogo; e PATHOS (IA) - o que sofre, sofredor; doença" (Paulo, 2001). Em outras palavras, a homeopatia é a terapia que busca estimular o organismo doente, cuja escolha da medicação irá variar de paciente e de situações (Pires, 2005). Essa terapia opta por medicar cada animal de maneira única, considerando cada estrutura, formas e particularidades do paciente (Braccini et al., 2019). Os fundamentos da homeopatia são bem descritos por Hahnemann em seu livro: "Órganon da arte de curar" (Santos, 2014).

Essa forma alternativa de tratamento, diferentemente da alopatia convencional, individualiza o animal, sendo uma melhor forma de curá-lo e ajudá-lo, por este motivo, a homeopatia vem crescendo cada vez mais na clínica de pequenos animais. Invariavelmente, o tutor busca esse tipo de terapia quando a alopatia não tem mais resultado, tentando, de alguma forma, minimizar ou findar a dor e/ou a doença do seu animal ( Souza, 2002).

\section{Doença renal crônica (DRC)}

Uma patologia de grande relevância na clínica de pequenos animais, em especial os senis, é a insuficiência renal, cuja evolução varia de acordo com o grau de lesão estrutural e funcional do parênquima renal. Quando há possibilidade de reversão dos danos na função renal, caracteriza-se como insuficiência renal aguda. Todavia, quando há irreversibilidade no processo, temos a chamada doença renal crônica (Brown et al., 1997; Polzin et al., 2005). Além da irreversibilidade, observa-se uma taxa de filtração glomerular (TFG) de até 50\% em relação ao seu normal (Brown et al., 1997; Polzin et al., 2005).

De forma a permitir um correto conhecimento da evolução da lesão renal, A International Renal Interst Socity (IRIS) desenvolveu uma classificação para doença renal crônica (DRC) em cães. Pacientes com enfermidades que predispõem a DRC, sem alterações clínicas e laboratoriais, são considerados no 
estádio 0 . No estádio 1 , a creatinina sérica está inferior a $1,4 \mathrm{mg} / \mathrm{dL}$, a poliúria e polidipsia podem estar presentes, bem como a incapacidade de concentrar urina. A creatinina sérica entre $1,4 \mathrm{mg} / \mathrm{dL}$ e 2,0 mg/dL, com manifestação clínica de poliúria e polidipsia, são características dos pacientes no estádio 2. No estádio 3, a creatinina sérica se comporta entre $2,1 \mathrm{mg} / \mathrm{dL}$ a 5,0 mg/dL, com manifestações clínicas moderadas da síndrome urêmica. No estádio 4, a creatinina sérica está superior a 5,0 $\mathrm{mg} / \mathrm{dL}$, com manifestações clínicas graves da síndrome urêmica, como alterações gastrointestinais, neuromusculares e cardiovasculares (Dalton, 2011).

Ainda na classificação proposta pela IRIS, existem sub-estágios relacionados à proteinúria renal e a hipertensão arterial sistêmica, considerados como fatores independentes de progressão da DRC (Bacic et al., 2010; Grauer, 2007; Waki et al., 2010). Na sequência serão apresentados, de forma detalhada, os sinais clínicos evidentes em pacientes classificados no estágio 4 da DRC e tratamentos alopáticos de eleição.

\section{Uso da homeopatia em doentes renais}

Animais acometidos pela Doença Renal Crônica podem apresentar uma série de manifestações clínicas, que variam de acordo com a idade do animal; velocidade da progressão; gravidade; espécie; presença de outra enfermidade não relacionada e administração concomitante de medicamentos. Esses fatores, associados à uremia incluem: polidpsia, anorexia, letargia, vômito, halitose com odor amoniacal, depressão, desidratação, hemorragia gastrointestinal, tremores musculares, convulsão, estomatites ulcerativas, coma, hipertensão e perda de peso (Gerber, 2017; Nelson \& Couto, 2015; Queiroz \& Fioravanti, 2014a; Rodriguez, 2012; Stockham \& Scott, 2011).

Em relação à glutamina, uma parte substancial de origem exógena é absorvida pelos enterócitos. Todavia, além da glutamina oriunda da dieta, o músculo esquelético (em grande parte) e os rins também produzem essa proteína de forma endógena. No rim, a glutaminase renal apresenta uma acentuada regulação pelo $\mathrm{pH}$, justificando a importante função renal de regular o $\mathrm{pH}$ sanguíneo. Não somente, a glutamina é fundamental na remoção de amônia no cérebro, impedindo a neurotoxicidade, uma vez que o grupamento amida proporciona uma reserva segura, não tóxica e transporta amônia. Esse mecanismo justifica a perda de massa muscular em animais doentes renais (Queiroz \& Fioravanti, 2014b).

Diante do exposto, um animal que se encontra em estágio avançado da DRC, apresentará toda a sintomatologia listada, uma vez que possui um número reduzido de néfrons, com comprometimento severo da função renal. $O$ tratamento alopático inclui o controle da desidratação com reposição e manutenção da fluidoterapia; o controle da hiperfosfatemia (garantindo que as concentrações de fósforo se mantenham inferiores a $6 \mathrm{mg} / \mathrm{dl}^{-1}$ tanto para cães quanto para gatos), o uso de bloqueadores de $\mathrm{H}_{2}$ (Famotidina), protetores de mucosa (Sucralfato), além de antieméticos (Maropitan, Ondansetrona) (Allen et al., 2000; Polzin, 2011; Waki et al., 2010).

Destaca-se ainda como tratamento a regulação da acidose metabólica, o uso da eritropoetina recombinante humana (50 a 100UI $/ \mathrm{kg}^{-1}$ duas a três vezes por semana) para o controle da anemia e a suplementação com ferro, em cães e gatos é de 100 a $300 \mathrm{mg} \mathrm{dia}^{-1}$ e de 50 a $100 \mathrm{mg} \mathrm{dia}^{-1}$, respectivamente (Waki et al., 2010).

De forma racional, é preciso compreender que nesta fase os néfrons remanescentes são incapazes de garantir e recuperar a função renal, sendo todos os procedimentos de ganho temporário e não com o fim de levar à cura e a resolução de possível sofrimento por parte do animal. A solução cabível seria o transplante do órgão, porém, devido à baixa sobrevida observada, a terapia de imunossupressão póstransplante, a predisposição ao desenvolvimento de infecções e linfomas e ao aspecto ético sobre o doador, faz dessa possibilidade uma realidade distante (Adin et al., 2001; Bernsteen et al., 2000; Katayama, 2002).

Em relato de caso, Bruno et al. (2018) demonstraram que uma cadela com valores elevados de creatina e ureia, hipertermia e mialgia em membros pélvicos à palpação, ptialismo e desidratação em exame físico apresentou melhora significativa nos quadros de êmese, ataxia, mioclonia de membros pélvicos, algia, hiporexia, oligodipsia e apatia após 5 dias de tratamento homeopático. A terapia prescrita foi Arnica Montana 30cH e Carduus marianus $6 \mathrm{cH}$ duas gotas ao dia, por 20 dias e/ou ao demonstrar dor. O relato de caso concluiu enaltecendo a melhora na qualidade de vida e a diminuição de risco para o paciente (Bruno et al., 2018). 
Diante do exposto, fica claro que as diretrizes alopáticas para tratamento de doentes renais crônicos em estágio final, apesar de bem estabelecidas, não garantem a reversibilidade da função renal, tampouco garante o alívio de eventual dor e sofrimento ao paciente. Dessa forma, a homeopatia se apresenta como uma alternativa de tratamento paliativo, a fim de garantir a diminuição de riscos ao paciente e a melhora de sua qualidade de vida.

\section{Referências bibliográficas}

Adin, C. A., Gregory, C. R., Kyles, A. E., \& Cowgill, L. (2001). Diagnostic predictors of complications and survival after renal transplantation in cats. Veterinary Surgery, 30(6), 515-521. https://doi.org/10.1053/jvet.2001.28418.

Allen, T. A., Polzin, D. J., \& Adams, L. G. (2000). Renal disease. In M. S. Hand, C. D. Thatcher, R. L. Remillard, \& P. Roudebush (Eds.), Small animal clinical nutrition (pp. 563-604). Mark Morris Institute.

Bacic, A., Kogika, M. M., Barbaro, K. C., Iuamoto, C. S., Simões, D. M. N., \& Santoro, M. L. (2010). Evaluation of albuminuria and its relationship with blood pressure in dogs with chronic kidney disease. Veterinary Clinical Pathology, 39(2), 203-209. https://doi.org/10.1111/j.1939165X.2009.00207.x.

Bear, J., \& Bellucco, W. (2006). Florais de Bach: O livro das fórmulas. Editora Pensamento.

Bernsteen, L., Gregory, C. R., Kyles, A. E., Wooldridge, J. D., \& Valverde, C. R. (2000). Renal transplantation in cats. Clinical Techniques in Small Animal Practice, 15(1), 40-45.

Braccini, G. L., Casetta, J., Silva, S. C. C., Carniatto, C. H. O., Santos, V. D. R., \& Costa, V. F. (2019). Aplicação da homeopatia na produção animal. Revista Valore, 4, 310-323.

Brown, S. A., Crowell, W. A., Brown, C. A., Barsanti, J. A., \& Finco, D. R. (1997). Pathophysiology and management of progressive renaldisease. The Veterinary Journal, 154(2), 93-109. https://doi.org/10.1016/S1090-0233(97)80048-2.

Bruno, F., Feitosa, C. B., \& Coelho, C. P. (2018). Abordagem homeopática em cão jovem com insuficiência renal crônica: relato de caso. Revista de Educação Continuada em Medicina Veterinária e Zootecnia Do CRMV-SP, 16(2), 78-79.

Costa, N. C., Araújo, R. L., \& Freitas, G. B. L. (2009). Homeopatia: Um campo terapêutico fundamental no cuidado veterinário de animais de produção. Revista Salus, 3(2), 73-89.

Dalton, R. N. (2011). Creatinina sérica e taxa de filtração glomerular: percepção e realidade. Jornal Brasileiro de Patologia e Medicina Laboratorial, 47(1), 8-11. https://doi.org/10.1590/s167624442011000100001.

Gerber, F. (2017). Diagnóstico e estadiamento da doença renal crônica em cães. Revista Científica de Medicina Veterinária do UNICEPLAC, 4(2), 46-60.

Grauer, G. F. (2007). Measurement, interpretation, and implications of proteinuria and albuminuria. Veterinary Clinics of North America: Small Animal Practice, 37(2), 283-295.

Katayama, M. (2002). Renal transplantation in cats: techniques, complications, and immunosuppression. Compendium on Continuing Education for the Practicing Veterinarian, 24(11), 874-882.

Nelson, R. W., \& Couto, C. G. (2015). Medicina interna de pequenos animais (Issue 1). Elsevier Editora.

Paulo, A. L. D. (2001). O que você precisa saber sobre o Medicamento Homeopático. In $O$ que você precisa saber sobre o medicamento homeopatico (p. 124). Organon.

Pereira, A. I. S. (2012). A abordagem homeopática aplicada na prática clínica veterinária: um estudo retrospectivo.

Pires, M. (2005). A homeopatia para os animais. Embrapa Gado de Leite-Comunicado Técnico, 46, 1-4.

Polzin, D J, Osborne, C. A., \& Ross, S. (2005). Chronic Kidney Disease. In S. J. Ettinger \& E. C. Feldman (Eds.), Textbook of veterinary internal medicine (pp. 1756-1785). Elsevier Saunders.

Polzin, David J. (2011). Chronic kidney disease in small animals. Veterinary Clinics: Small Animal Practice, 41(1), 15-30. 
Queiroz, L. L., \& Fioravanti, M. C. S. (2014a). Distúrbios endócrinos na doença renal crônica em cães. Enciclopédia Biosfera, 10(18), 1326-1344.

Queiroz, L. L., \& Fioravanti, M. C. S. (2014b). Tratamento da doença renal crônica em pequenos animais: um guia para o médico veterinário. Enciclopédia Biosfera, 10(18), 1-33.

Rodriguez, O. C. (2012). Estadiamento e tratamento da doença renal crônica. In O. Cortadelas (Ed.), Manual de nefrologia e urologia clinica canina e felina (pp. 161-177). Medvet.

Santos, R. (2014). Homeopatia: histórico e fundamentos. Revista Científica da Faculdade de Educação e Meio Ambiente, 5, 60-78.

Souza, M. F. A. (2002). Homeopatia veterinária. Conferência Virtual Global Sobre Produção Orgânica de Bovinos de Corte, 1, 1-4.

Stockham, S. L., \& Scott, M. A. (2011). Fundamentos de patologia clínica veterinária. In Guanabara Koogan (Vol. 8).

Waki, M. F., Ribas Martorelli, C., Erdmann Mosko, P., \& Kogika, M. M. (2010). Classificação em estágios da doença renal crônica em cães e gatos: abordagem clínica, laboratorial e terapêutica. Ciência Rural, 40(10), 1-9.

Histórico do artigo:

Recebido: 8 de julho de 2021

Aprovado: 28 de agosto de 2021

Disponível on-line: 11 de novembro de 2021
Licenciamento: Este artigo é publicado na modalidade Acesso Aberto sob a licença Creative Commons Atribuição 4.0 (CC-BY 4.0), a qual permite uso irrestrito, distribuição, reprodução em qualquer meio, desde que $\mathrm{o}$ autor e a fonte sejam devidamente creditados. 